\title{
Tracheoesophageal fistula managed with tracheal stent through flexible bronchoscopy without fluoroscopy
}

\author{
G. Stratakos'1, C. Zisis², I. Bellenis², V. Filaditaki1, A. Liapikou1, \\ S. Zakynthinos ${ }^{1}$, C. Roussos ${ }^{1}$
}

ABSTRACT: Tracheoesophageal fistula managed with tracheal stent through flexible bronchoscopy without fluoroscopy. G. Stratakos, C. Zisis, I. Bellenis, V. Filaditaki, A. Liapikou, S. Zakynthinos, C. Roussos.

Inoperable malignant tracheoesophageal fistula (TEF) is characterised by an extremely poor prognosis. Tracheal or double (tracheal-esophageal) stenting through rigid bronchoscopy has been suggested as a valuable therapeutic option.

We report on a patient with a large TEF successfully sealed by deployment of a self-expandable stent through flexible bronchoscopy (FB) without fluoroscopy. Dramatically improved health status permitted him to undergo radiation, attaining further clinical improvement. Four months after stent placement no sequelae were observed. During the fifth month a new fistula developed distally to the stent finally leading to death from septic complication.

Palliative management of inoperable malignant TEF by tracheal stent placement through FB without fluoroscopy, is feasible, safe and rewarding leading to important clinical improvement.

Monaldi Arch Chest Dis 2006; 65: 4, 225-227.

Keywords: Tracheoesophageal fistula, flexible bronchoscopy, auto-expandable airway stents, interventional pulmonology.

1 Critical Care and Pulmonary Medicine Division, University of Athens,

2 Thoracic Surgery Department, "Evaggelismos” Hospital, Athens, Greece.

Correspondence: Grigoris Stratakos MD, Critical Care and Respiratory Division, "Evaggelismos" Hospital; $45-47$ Ipsilandou str, 10675 Athens, Greece; e-mail: grstrat@hotmail.com

Inoperable esophageal cancer is characterised by an extremely poor prognosis especially when tracheoesophageal fistula (TEF) develops. Death from aspiration pneumonias and sepsis can be expected within days unless fistula can be sealed [1].

Oesophageal stenting with the intention of sealing fistulas between alimentary and respiratory tract, when feasible, is often insufficient and noneffective $[1,2]$. Instead, tracheal or double (tracheal-oesophageal) stenting has been suggested as a valuable option [1-3]. Deployment of various types of airway stents through rigid bronchoscopy or more recently flexible bronchoscopy (FB) under fluoroscopic guidance, have been reported to effectively seal TEF, restore airway patency and achieve median survival of $110 \pm 30$ days at best $[1,3,4]$.

Although FB is advantageous compared with the rigid technique as general anesthesia and mechanical ventilation are not necessary, fluoroscopy requires specialised equipment and staff, exposes the patient and staff to radiation and increases the time and cost of the procedure [5]. Two studies however, have recently advocated the efficacy and safety of bronchoscopic balloon dilatation and self-expandable stent deployment without fluoroscopy $[6,7]$.

We report on a patient with a large malignant TEF who was successfully treated with tracheal stenting by flexible bronchoscopy without fluoroscopic guidance.

\section{Case Report}

A 52 year old male with primary oesophageal cancer invading the mediastinum and a large tracheoesophageal fistula causing dysphagia, cachexia and recurrent lung infections due to aspiration, was referred to "Evaggelismos" tertiary-care university hospital.

Upon admission the patient was exhausted, with a temperature of $38.5^{\circ} \mathrm{C}$, tachycardia (110 beats/min), tachypnea [22 breaths/min) and oxygen saturation $\left(\mathrm{SPO}_{2}\right)$ of $90 \%$ while breathing room air. Auscultation revealed crepitation rales in both lungs.

The lesion was evaluated by thoracic surgeons as being inoperable due to its extensive mediastinal invasion. An oesophageal endoscopy revealed complete obstruction of the oesophageal lumen which did not permit the passage of any instrument or guide-wire. A gastrostomy was performed to bypass the fistula and allow for patient alimentation. After interdisciplinary counseling, interventional bronchoscopic management of the airways was encountered while general anesthesia and rigid bronchoscopy were judged to be far too dangerous given the critical status of the patient. 
A bronchoscopy was performed with a flexible video-bronchoscope disposing a wide working channel (BF XT 160; Olympus; Melville NY). Premedication included $1 \mathrm{mg}$ atropine i.m, local lidocaine and $2 \mathrm{mg}$ midazolam i.v. During examination, a large TEF (figure 1) at the left posterior side of mid-trachea could be observed. The length of the abnormal communication was measured, by withdrawing the bronchoscope from the distal to the proximal end of the lesion, to be approximately $40 \mathrm{~mm}$. No lumen obstruction was evident throughout the whole length of the trachea. Abundant purulent secretions could pass back and forth through the fistula and flooded the whole tracheobronchial tree. A guide wire was introduced in the trachea distally to the TEF, the bronchoscope was removed and a stent (Silmet ${ }^{\circledR}$ Novatech $16 \mathrm{~mm} \mathrm{x}$ $60 \mathrm{~mm}$ ) mounted on an insertion catheter was placed over the guide wire. The bronchoscope was then reintroduced to ascertain direct visualisation of the stent catheter. The catheter was advanced until the middle of the stent was located adjacent to the fistula and then it was gradually deployed. After full expansion of the mesh, the introduction catheter was slowly retracted together with the guide wire from within the stent.

Successful sealing of the tracheoesophageal fistula and restoration of the airway patency was achieved (figure 2). A thorough bronchial toilet was performed leading to immediate increase in $\mathrm{SPO}_{2}$. Two days later, the patient experienced important relief of his symptoms and was discharged. Improved health status permitted him to undergo radiation for the mediastinal mass on an ambulatory basis.

We followed up this patient by means of clinical examination and bronchoscopy once a month. Four months after intervention, he remained in a good condition without sequelae. He had gained $6 \mathrm{~kg}$ of body weight and did not complain of any important symptoms. $\mathrm{SPO}_{2}$ was $97 \%$ on room air and auscultation was normal. A stent remained in place, effectively sealing the TEF.

During the fifth month of follow-up, the patient complained of a persistent cough and purulent expectoration. A bronchoscopic examination this time revealed development of a new fistula distally to the lower extremity of the stent (adjacent to the tracheal carina) not amenable to further treatment. A week later this patient was re-admitted with bilateral pneumonia and died soon after from septic shock on the 132 day after initial intervention.

\section{Discussion}

Sole oesophageal stenting in cases of TEF, has been shown to initially improve the quality of life but usually fails to seal the fistula sufficiently and may often produce extrinsic tracheal compression. Simultaneous stenting of both trachea and oesophagus or tracheal stenting in case of complete oesophageal obstruction, yields the best clinical results and has become a standard procedure $[1,3$, 8]. Although various types of tracheal stents in-

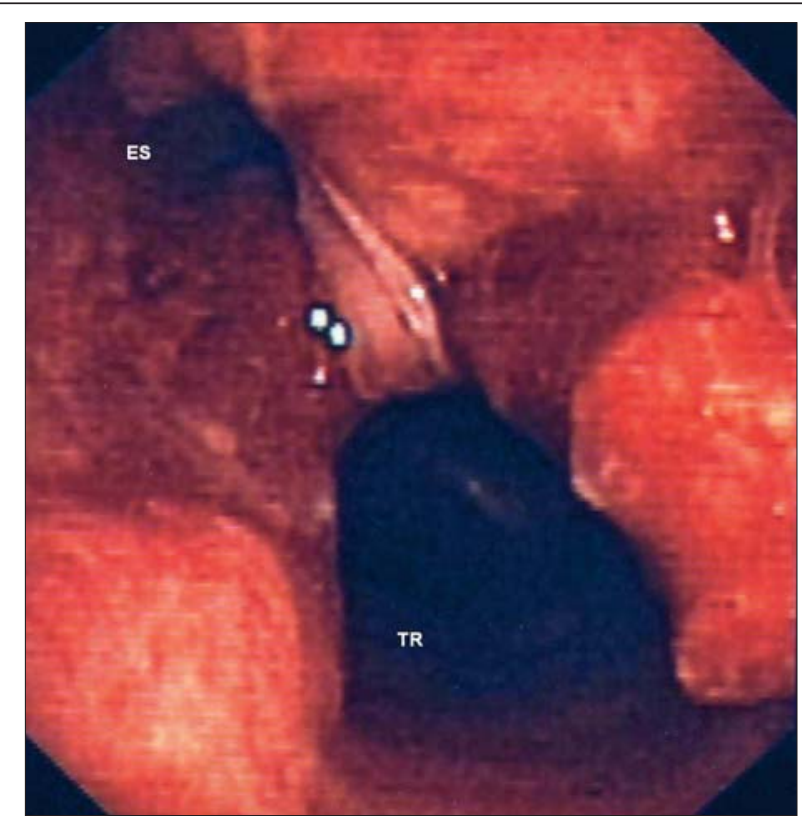

Fig. 1. - A huge tracheoesophageal fistula can be observed at the left posterior side of mid-trachea. The overall length of abnormal communication between oesophagus (Es) and trachea (TR) was measured at approximately $40 \mathrm{~mm}$. Distal trachea was otherwise normal.

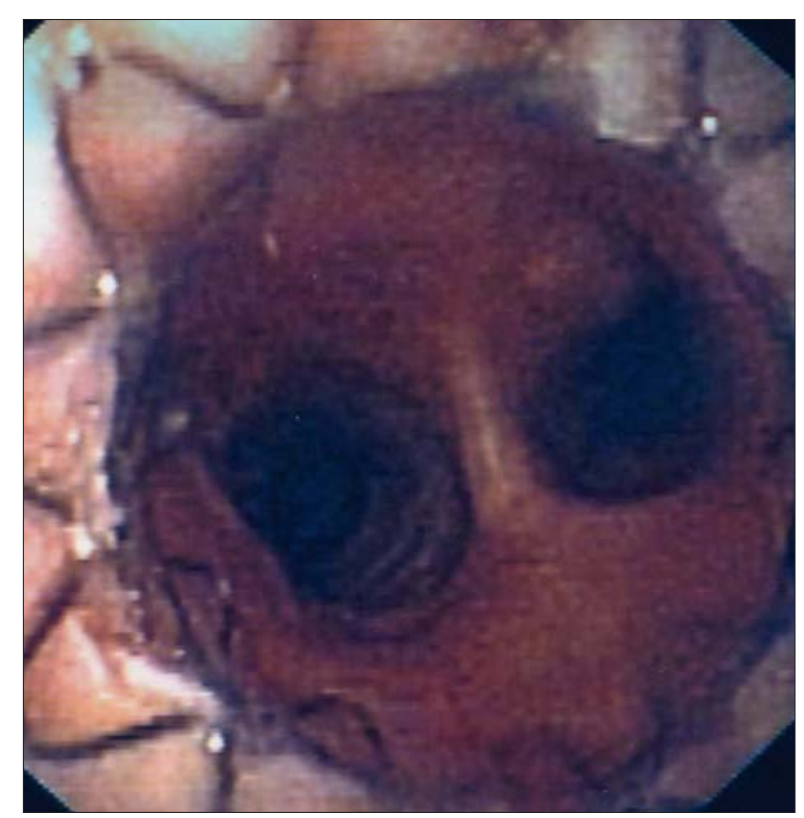

Fig. 2. - After complete stent deployment the fistula has been sealed and the airway lumen has been restored. The distal edge of the prosthesis is situated few millimeters above the main carina.

cluding Dumon silicone, Dynamic Y shaped and covered auto-expandable metallic stents have been reported to give good results in cases of TEF, we would opt for auto-expandable stents because of their flexibility and better conforming to the irregular airways we usually encounter in a TEF. Homogeneous radial force exerted to the tracheal wall from the mesh of the stent, prevents it from migrating and ensures adequate sealing of the fistula.

Most experts prefer to place stents through rigid bronchoscope under general anesthesia as it is both safer and more comfortable [8]. However, the paucity of available room and anesthetists in the operation block of most hospitals, has prompt- 
ed respiratory physicians to develop less "invasive" techniques and use flexible bronchoscope to place auto-expandable stents, under fluoroscopic guidance $[4,5]$. This approach does not require general anesthesia, can be held in a normal bronchoscopy suite, is efficient and safe and may even prove less aggressive or traumatic to the airway wall. No severe complications have been reported so far in large series [5,6]. Should removal of the stent be necessary, rigid bronchoscope and standard interventional techniques will of course be warranted [9].

Recommendation for fluoroscopic guidance for metal stent insertion has been questioned recently $[6,7]$. However, to the best of the authors' knowledge there has been no previous report for the deployment of a self-expanding stent for TEF management with flexible bronchoscopy without fluoroscopy. Herth et al. [6] reported 10 cases of TEF which have been seen to have been treated by tracheal stent insertion not clarifying whether rigid or flexible bronchoscopy had been employed. If any tracheal lumen obstruction was evident, prior to stenting, additional dilatation techniques including balloon or rigid scope dilatation should have been applied.

The Silmet covered stent used in this case, is a modern self-expanding device made of nitinol metal alloy characterised by its thermal shape memory. It is covered in its outer surface by an ultra thin polyester membrane not allowing for malignant tissue growth in the airway lumen. The final $5 \mathrm{~mm}$ on each edge remain uncovered permitting embedment of the stent in the airway mucosa, leading to additional stability and smaller migration rate. Other covered auto-expandable stents as Ultraflex ${ }^{\circledR}$ or Alveolus ${ }^{\circledR}$ could have equally been used using similar technique. As no comparative study has shown any special superiority of one against another, selection of the type of covered auto-expandable stent is carried out on the basis of previous experience, personal preferences as well as local availability.

It is necessary to emphasise, however, that even if the approach used in this case simplifies the insertion of this particular type of stent not requiring general anesthesia or specialised fluoroscopic equipment, a plethora of other stents as well as most complications and eventual removal of the stent, exclusively need rigid bronchoscopy to be handled $[8,9]$. Thus even if less interventional techniques are chosen in selected cases, it is of utmost importance for an interventional pulmonologist to be trained and familiar with rigid bronchoscope and all related procedures.

A Y- stent could have possibly been a valuable option for the second fistula developed in the patient. However, in that case, the patient should have undergone removal of the first stent through rigid bronchoscopy and general anesthesia, before the new stent was put in place. This procedure could be highly dangerous and of questionable benefit given his clinical status and the extent of the disease.

In conclusion we report the successful management of a patient with inoperable malignant TEF treated with self-expandable tracheal stent using flexible bronchoscopy without fluoroscopy. This technique is relatively simple, safe and rewarding.

\section{References}

1. Freitag L, Tekolf E, Steveling H, Donovan TJ, et al Management of malignant esophagotracheal fistulas with airway stenting and double stenting. Chest 1996; 110: 1155-60.

2. Belleguic C, Lena H, Briens E, et al. Tracheobronchial stenting in patients with esophageal cancer involving the central airways. Endoscopy 1999; 31: 232-6.

3. Colt HG, Meric B, Dumon JF. Double stents for carcinoma of the esophagus invading the tracheobronchial tree. Gastrointest Endosc 1992; 38: 485-89.

4. Dasgupta A, Dolmatch BL, Abi-Saleh WJ, et al. Selfexpandable metallic airway stent insertion employing flexible bronchoscopy. Chest 1998; 114: 106-109.

5. Saad CP, Murthy S, Krizmanich G, et al. Self-expandable metallic airway stents and flexible bronchoscopy. Long term outcomes analysis. Chest 2003; 124: 19931999.

6. Herth F, Becker HD, LoCicero J, et al. Successful bronchoscopic placement of tracheobronchial stents without fluoroscopy. Chest 2001; 119: 1910-1912.

7. Mayse ML, Greenheck J, Friedman M, et al. Successful balloon dilatation of non-malignant tracheobronchial obstruction without fluoroscopy. Chest 2004; 126: 634637.

8. Freitag L. Tracheobronchial stents. In Bolliger CT, Mathur PN (Eds): Interventional Bronchoscopy. Prog Respir Res. Basel, Karger, 2000, vol 30. pp 171-186.

9. Noppen M, Stratakos G, D'Haese J, et al. Removal of covered self-expandable metallic airway stents in benign disorders. Indications, Technique and outcomes. Chest 2005; 127: 482-487. 